\title{
Vortex Knot Cascade in Polynomial Skein Relations
}

\author{
Renzo L. Ricca ${ }^{1, a)}$ \\ ${ }^{1}$ Department of Mathematics $\mathcal{E}$ Applications, U. Milano-Bicocca, Via Cozzi 55, 20125 Milano, Italy \\ a)Corresponding author: renzo.ricca@unimib.it \\ URL: http://www.matapp.unimib.it/ ricca
}

\begin{abstract}
The process of vortex cascade through continuous reduction of topological complexity by stepwise unlinking, that has been observed experimentally in the production of vortex knots (Kleckner \& Irvine, 2013), is shown to be reproduced in the branching of the skein relations of knot polynomials (Liu \& Ricca, 2015) used to identify topological complexity of vortex systems. This observation can be usefully exploited for predictions of energy-complexity estimates for fluid flows.
\end{abstract}

\section{HOMFLYPT POLYNOMIAL FOR VORTEX KNOTS}

Tangles of vortex filaments forming an intricate networks of thin tubes are generic features of quantum turbulence in superfluids $[1,2]$ and under appropriate circumstances characterize the decay of vortical flows in classical turbulence $[3,4]$. These filaments may form knots and links, that decay rapidly through reconnections to form various loops, till final dissipation. Kinetic helicity is an appropriate measure of topological complexity [5, 6], but it is known to suffer from some limitations (see, in particular, Sec. 6 of [7] for comments). To overcome these difficulties Liu \& Ricca $[7,8,9]$ have successfully derived various knot polynomials (Kauffman bracket, Alexander-Conway, Jones and HOMFLYPT) for fluid knots as new invariants of ideal fluid mechanics. By applying knot theoretical techniques to vortex flows, in particular, the HOMFLYPT polynomial has been derived from the kinetic helicity $H$ of vortex knots.

Theorem 1 (Liu \& Ricca, 2015) Let $K$ denote a physical knot. If the helicity of $K$ is $H=H(K)$, then

$$
e^{H(K)}=e^{\oint_{K} \mathbf{u} \cdot \mathbf{d} \mathbf{l}},
$$

appropriately re-scaled, satisfies (with a plausible statistical hypothesis) the skein relations of the HOMFLYPT polynomial $P=P_{K}$.

Remark 1. If $K$ is a vortex knot, then $\mathbf{u}$ is the self-induced velocity associated with its vorticity, aligned along the knot centerline of elementary line element dl. To make sense of $e^{H(K)}, H(K)$ must be normalized with respect to some reference value of vortex circulation $\Gamma$.

Remark 2. The derivation of the result above relies on a statistical hypothesis, that assumes equally probable state decomposition of the crossing sites in the minimal knot diagram. This is equivalent to the ergodic assumption that all possible (virtual) reconfiguration states of the given knot or link are equally admissible (see [9] for further explanation).

Remark 3. The skein relations of the HOMFLYPT polynomial $P=P_{K}$ are given by:

(i) $\quad P(\bigcirc)=1$,

(ii) $\quad a P(\nearrow)-a^{-1} P(\nearrow)=z P(\nearrow)$,

with $z=k-k^{-1}$ and

$$
\left.\begin{array}{r}
k=e^{\bar{\omega}}, \quad \bar{\omega}=2 \lambda_{\omega} \bar{H}(\bigcirc) \\
a=e^{\bar{\tau}}, \quad \bar{\tau}=\lambda_{\tau} \bar{H}(\bigodot)
\end{array}\right\} \quad\left\{\lambda_{\omega}, \lambda_{\tau}\right\} \in[0,1],
$$


where $\bar{H}(C \bigcirc)$ and $\bar{H}(\Theta)$ denote respectively writhe and twist helicity of a reference configuration, and $\lambda_{\omega}$ and $\lambda_{\tau}$ are parameters that take into account the uncertainty (or probability) associated with these values.

The first skein relation (2) states that the value $P$ of the unknot (or any topologically trivial loop) is 1 ; the second relation (3) states that the difference between the values of $P$ calculated for two identical knots in their minimal projection, weighted by their respective coefficients and of opposite sign at a given crossing, is equal to the value of $P$ calculated for the same knot diagram, whose given crossing has been replaced by a non-crossing ("smoothing"). Examples of polynomial computations based on the recursive application of the skein relations above can be readily found by some of the knot tabulators available on-line (such as KnotAtlas [10]).

Since each knot carries information about its own circulation $\Gamma$, that is a conserved quantity under ideal evolution, and $H(K)$ is also an invariant of ideal fluid mechanics, then $P_{K}$ is a diffeomorphic invariant of knot type and circulation, thus providing a new topological invariant of ideal fluid flows [9]. $P_{K}$ provides a new tool to investigate structural complexity aspects of complex systems, and it can be used to investigate relations between energy transfers and complexity. In what follows we show that indeed the skein relations (2)-(3) above can be used to detect topological transitions associated with dissipation.

\section{STEPWISE UNLINKING IN THE POLYNOMIAL SKEIN RELATIONS}

Real vortex knots and links have been produced in water by recent laboratory experiments [11]. In presence of viscosity structural reconnections take place, inducing a continuous topological change of vorticity. In general, when two disjoint vortex structures reconnect the result is a single, closed vortex, and when a single closed vortex reconnects with itself the result is two closed loops. The change in topology of vortex structures can be detected by knot polynomials. In the above experiments [11] trefoil knots and Hopf links are observed to follow a reconnection cascade that leads to a consistent reduction of topological complexity in steps of one crossing lost at each reconnection event, till final dissipation of unlinked, unknotted smaller loops. According to [11], the cascade process is schematically reproduced by the sequence (see the diagrams in Fig. 1)

$$
\boldsymbol{T}^{L} \rightsquigarrow \boldsymbol{H}^{-} \rightsquigarrow \boldsymbol{U}_{1 \propto}^{1} \rightsquigarrow \boldsymbol{U}^{2},
$$

where a left-handed trefoil knot $\boldsymbol{T}^{L}$ first reconnects to form a negative Hopf link $\boldsymbol{H}^{-}$, then a 1-fold, unknotted loop $\boldsymbol{U}_{1 \propto}^{1}$, a small pair of unfolded, unknotted, unlinked loops $\boldsymbol{U}^{2}$, till the final decay to unstructured, small-scale turbulence. Note the presence of the apparent crossing in the folding of $\boldsymbol{U}_{1 \propto}^{1}$, responsible for the transition to $\boldsymbol{U}^{2}$. Remarkably, this cascade process governed by anti-parallel reconnection of vortex strands can be reproduced by the branching of the skein relation (3). As prescribed by equation (3), a negative crossing is switched into a positive crossing and a "smoothing" of the same crossing into two parallel strands; by re-writing eq. (3) as

$$
P(\nearrow)=a^{2} P(\nearrow)-a z P()()
$$

we can see how direct application of this equation to the left-hand trefoil knot $\boldsymbol{T}^{L}$ of Fig. 1 generates (a) the unknot $\boldsymbol{U}_{3 \propto}^{1}$, and (b) the Hopf link $\boldsymbol{H}^{-}$. By recursive selection of the smoothing process applied to the encircled crossing site, we obtain the sequence (5), observed experimentally in [11].

Under the assumption that topological complexity gets reduced by stepwise unlinking, Shimokawa et al. [12] prove that such a cascade process is unique. The proof relies on topological information about $(2,2 n+1)$ torus knots and $(2,2 n)$ torus links, and it is not known if it holds true for more general knot and link types. For instance, it would be interesting to test the cascade process associated with the generation of the Borromean rings (a 3-component link), given by the standard minimal projection ( 6 crossings). If we assumed that a first reconnection took place in the outer region of $\boldsymbol{B}$, a simple exercise would show that the following cascade could be expected:

$$
\boldsymbol{B} \rightsquigarrow \tilde{\boldsymbol{W}} \rightsquigarrow \boldsymbol{T}_{1 \propto}^{R} \rightsquigarrow \boldsymbol{T}^{R} \sqcup \boldsymbol{U}^{1} \rightsquigarrow \boldsymbol{H}^{+} \sqcup \boldsymbol{U}^{1} \rightsquigarrow \boldsymbol{U}_{1 \propto}^{1} \sqcup \boldsymbol{U}^{1} \rightsquigarrow \boldsymbol{U}^{3},
$$

where $\tilde{\boldsymbol{W}}$ denotes the mirror image of the standard Whitehead link (5 crossings), $\boldsymbol{T}_{1 \propto}^{R}$ the 1-fold, right-hand trefoil knot, $\boldsymbol{U}^{3} \equiv \boldsymbol{U}^{1} \sqcup \boldsymbol{U}^{1} \sqcup \boldsymbol{U}^{1}$ the disjoint union of 3 standard, unfolded, unknotted, unlinked loops ( $\sqcup$ standing for disjoint union of components). On the other hand, if a first reconnection took place in the inner region of $\boldsymbol{B}$, we could expect the alternative cascade:

$$
\boldsymbol{B} \rightsquigarrow \boldsymbol{W} \rightsquigarrow \boldsymbol{F}^{8} \rightsquigarrow \boldsymbol{H}_{1 \propto}^{-} \rightsquigarrow \boldsymbol{H}^{-} \sqcup \boldsymbol{U}^{1} \rightsquigarrow \boldsymbol{U}_{1 \propto}^{1} \sqcup \boldsymbol{U}^{1} \rightsquigarrow \boldsymbol{U}^{3},
$$




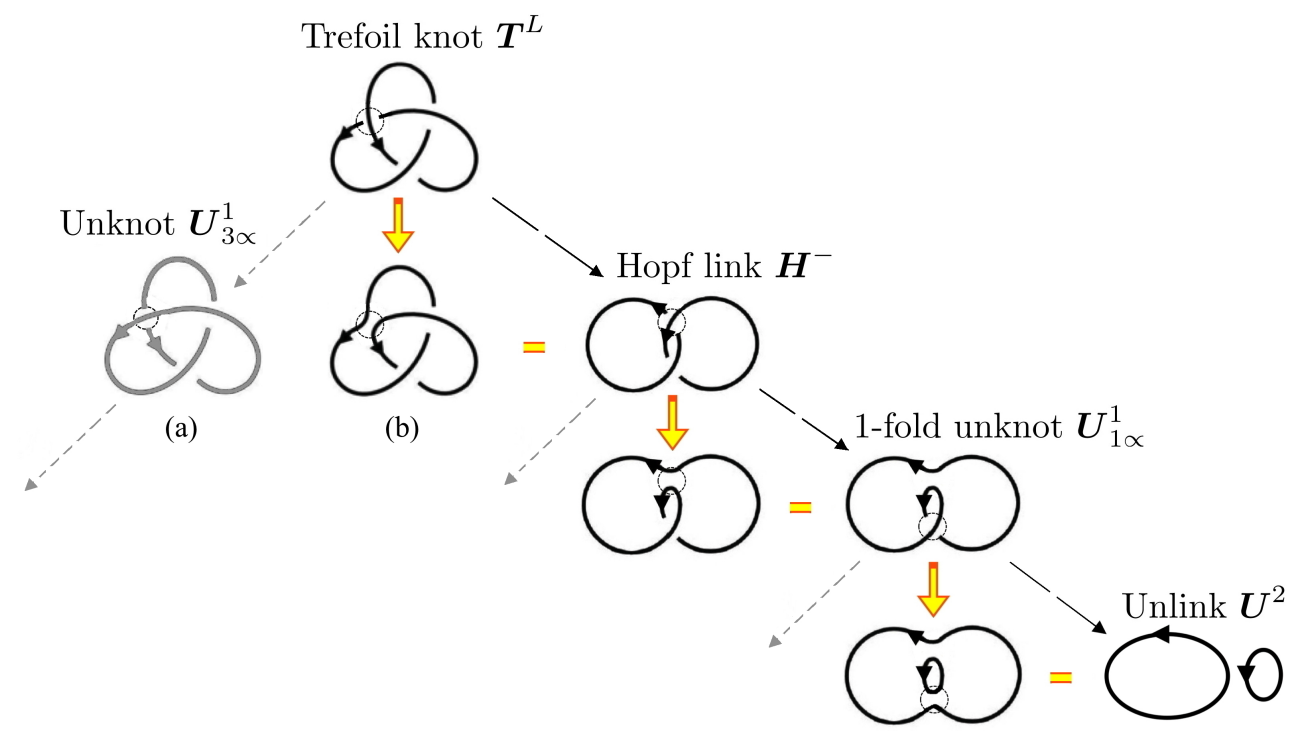

FIGURE 1. Reduction of topological complexity through stepwise unlinking in the mathematical reduction scheme associated with the recursive application of the polynomial skein relation (6). Note that the branching process, associated with the "smoothing" of the selected crossing (in evidence on the right), first generates (b) the standard Hopf link $\boldsymbol{H}^{-}$, and then the cascade (5) as obtained experimentally in [11].

where $\boldsymbol{W}$ denotes the standard Whitehead link, $\boldsymbol{F}^{8}$ the figure-of-eight knot and $\boldsymbol{H}_{1 \propto}^{-}$the 1-fold, negative Hopf link. Since the two processes above entail the same number of reconnections and decay to the same end state given by 3 disjoint, standard loops, from an energetic viewpoint both processes result equally probable.

\section{TWO-SCALE REGIME IN THE DISSIPATION PROCESS}

Anti-parallel reconnection seems the generic (if not the only) mechanism that governs change of topology of vortex filaments. During reconnection kinetic helicity gets dissipated; topologically this means dissipation of mutual linking between separate structures, and dissipation of self-linking $H(K)$ carried by each individual vortex knot $K$. For thin filaments, $H(K)$ admits decomposition in terms of writhe and twist, according to the Călugăreanu-White theorem $[13,14]:$

$$
H(K)=\Gamma^{2} S L=\Gamma^{2}(W r+T w),
$$

where $\Gamma$ is vortex circulation, $W r$ is writhe and $T w(=T+N)$ is total twist, given by the sum of total torsion $T$ and intrinsic twist $N$ (for proper definitions see [14]). Writhe and total torsion are global geometric quantities associated with curvature and torsion of the vortex centerline in space, while intrinsic twist measures the winding of the vortex lines around and along the vortex centerline. $H(K)$ is thus given by the contributions due to $\bar{H}(\circlearrowleft)$ ) and $\bar{H}\left(C_{\text {) }}\right.$.

Experimental measurements [15] show that $\bar{H}(\bigcirc)$ remains essentially conserved through reconnection, a result that has been proven rigorously [16] under anti-parallel assumption. This means that only $\bar{H}$ (C) (given essentially by intrinsic twist) gets dissipated at the reconnection site. This occurs at a length scale $\delta$ of the order of the vortex core. Since during reconnection writhe is frozen, dissipation of writhe helicity (and mutual linking) must take place on the integral scale $L \gg \delta$ of the vortex. For classical vortex filaments it is reasonable to assume that

$$
\frac{L}{\delta} \approx \frac{D}{\eta} \sim\left(\frac{U D}{v}\right)^{3 / 4}=R e_{D}^{3 / 4}
$$

where $D$ is the integral scale of the ambient fluid, $\eta$ the typical length scale given by the ratio of viscosity $v$ and dissipation rate, $U$ a reference fluid velocity, and $R e_{D}$ the Reynolds number [17]. Consequently, the associated typical 
time scales are given by

$$
\frac{t_{L}}{t_{\delta}} \approx \frac{t_{D}}{t_{\eta}} \sim\left(\frac{U D}{v}\right)^{1 / 2}=R e_{D}^{1 / 2} .
$$

Clearly, as the Reynolds number increases, length and time scales get more separated; since twist helicity becomes dissipated in a much shorter time than writhe helicity, we may identify two different regimes, where either twist or writhe dissipation prevails. On time scales given by $t_{\delta}<t \ll t_{L}$, writhe changes are expected to be modest, whereas for $t=O\left(t_{L}\right)$ intrinsic twist remains almost invariant, and major changes in helicity are likely to be due to changes of writhe and total torsion (centerline helicity).

These considerations can be usefully applied to complement on-going work [18] on applications of structural complexity methods to the analysis of the energetics of complex fluid systems, and can be equally extended to physical and biological systems based on filament modeling. In primis DNA recombination (where stepwise unlinking due to topo-isomerase action was first analyzed) [12], but also plasma loops in solar physics [19], structural knotting and linking in crystallography [20], defect formation in laser melted materials [21], and Bose-Einstein condensates in superconductors [22].

\section{ACKNOWLEDGMENTS}

I am grateful to De Witt Sumners, who addressed my attention to the paper [12]. Financial support from PRIN Project 2010-2011 "Geometric and analytic theory of Hamiltonian systems in finite and infinite dimensions" is gratefully acknowledged.

\section{REFERENCES}

1. A. W. Baggaley, C. F. Barenghi, A. Shukurov and Y. A. Sergeev, Europhys. Letts. 98, 26002 (2012).

2. L. Kondaurova, V. L'vov, A. Pomyalov and I. Procaccia, Phys. Rev. B 89, 014502 (2014).

3. S. Douady, Y. Couder and M. E. Brachet, Phys. Rev. Lett. 67, 983-986 (1991).

4. H. Mouri, A. Hori and Y. Kawashimab, Phys. Fluids 19, 055101 (2007).

5. C. F. Barenghi, R. L. Ricca and D. C. Samuels, Physica D 157, 197-206 (2001).

6. R. L. Ricca, Geophys. Astrophys. Fluid Dyn. 107, 385-402 (2013).

7. X. Liu and R. L. Ricca, J Fluid Mech. 773, 34-48 (2015).

8. X. Liu and R. L. Ricca, J. Phys. A: Math. \& Theor. 45, 205501 (2012).

9. R. L. Ricca and X. Liu, Fluid Dyn. Res. 46, 061412 (2014).

10. KnotAtlas: http://katlas.math.toronto.edu/wiki/Main_Page

11. D. Kleckner and W. T. M. Irvine, Nature Physics 9, 253-258 (2013).

12. K. Shimokawa, K. Isihara, I. Grainge, D. J. Sherratt and M. Vazquez, PNAS USA 110, 20906-20911 (2013).

13. R. L. Ricca and H. K. Moffatt, "The helicity of a knotted vortex filament," in Topological Aspects of the Dynamics of Fluids and Plasmas edited by H. K. Moffatt et al. (Kluwer Acad. Publs., Dordrecht, 1992), pp. 225-236.

14. H. K. Moffatt and R. L. Ricca, Proc. Roy. Soc. A 439, 411-429 (1992).

15. M. W. Scheeler, D. Kleckner, D. Proment, G, L. Kindlmann, and W. T. M. Irvine, PNAS USA 111, 1535015355 (2014).

16. C. E. Laing, R. L. Ricca and De W. L. Sumners, Sci. Rep. 5, 9224 (2015).

17. U. Frisch, Turbulence: The Legacy of A.N: Kolmogorov. (Cambridge U. P., Cambridge, 1995), p. 91.

18. R. L. Ricca, "Structural complexity of vortex flows by diagram analysis and knot polynomials," in How Nature Works, Emergence, Complexity and Computation 5, edited by I. Zelinka et al. (Springer-Verlag, Heidelberg, 2014), pp. 81-100.

19. J. W. Cirtain, L. Golub, A. R. Winebarger, B. De Pontieu, K. Kobayashi, R. L. Moore, R. W. Walsh, K. E. Korreck, M. Weber, P. McCauley, A. Title, S. Kuzin and C. E. DeForest, Nature 493, 501-503 (2013).

20. S. Tanda, T. Tsuneta, Y. Okajima, K. Inagaki, K. Yamaya and N. Hatakenaka, Nature 417, 397-398 (2002).

21. S. Lugomer, Phys. Rev. B 54, 4488-4491 (1996).

22. D. Proment, M. Onorato and C. F. Barenghi, Phys. Rev. E 85, 036306 (2012). 